\title{
Adapting SYCL's SIMT Programming Paradigm for Accelerators via Program Reconstruction
}

\author{
Jiashu Wang, Xun Deng, Kai-Ting Amy Wang, Zichun Ye \\ Huawei Canada Research Centre \\ Markham, Canada \\ \{jiashu.wang,xun.deng,kai.ting.wang,zichun.ye\}@huawei.com
}

\begin{abstract}
We present an IR-to-IR Converter that is capable of converting from LLVM IR to Halide IR and MLIR's Affine Dialect to support generation of high performance SYCL kernel code [10] targeting accelerators with explicit memory hierarchy design. The converter performs program reconstruction to raise abstraction of the IR. Once the IR is raised to the level of Halide IR, AKG [2] can be leveraged to generate performant DaVinci code [2]. Alternatively, when the IR is raised to MLIR's Affine Dialect, existing MLIR Affine passes with minor modifications can be readily used. Subsequently, the IR is lowered back to LLVM IR through progressive lowering. LLVM's LLC is used to create the final binary for both cases. We extend upon SYCL's buffer, accessor and parallel_for abstractions to facilitate the IR raising process.
\end{abstract}

\section{CCS CONCEPTS}

- Software and its engineering $\rightarrow$ Source code generation; • Computer systems organization $\rightarrow$ Single instruction, multiple data.

\section{KEYWORDS}

SYCL, Program Reconstruction, Parallel Computing, Tiling, MLIR, LLVM

\section{ACM Reference Format:}

Jiashu Wang, Xun Deng, Kai-Ting Amy Wang, Zichun Ye. 2021. Adapting SYCL's SIMT Programming Paradigm for Accelerators via Program Reconstruction . In 50th International Conference on Parallel Processing Workshop (ICPP Workshops '21), August 9-12, 2021, Lemont, IL, USA. ACM, New York, NY, USA, 6 pages. https://doi.org/ $10.1145 / 3458744.3473354$

\section{INTRODUCTION}

SYCL is a cross-platform abstraction layer from Khronos Group that enables code for heterogeneous processors to be written using standard ISO C++ with host and kernel code for an application contained in the same source file. There are many SYCL implementations in development with possibly Intel's DPC++ being the most active one with hundreds of commits per week [6].

Permission to make digital or hard copies of all or part of this work for personal or classroom use is granted without fee provided that copies are not made or distributed for profit or commercial advantage and that copies bear this notice and the full citation on the first page. Copyrights for components of this work owned by others than ACM must be honored. Abstracting with credit is permitted. To copy otherwise, or republish, to post on servers or to redistribute to lists, requires prior specific permission and/or a fee. Request permissions from permissions@acm.org.

ICPP Workshops '21, August 9-12, 2021, Lemont, IL, USA

(c) 2021 Association for Computing Machinery.

ACM ISBN 978-1-4503-8441-4/21/08 . \$ $\$ 15.00$

https://doi.org/10.1145/3458744.3473354
Since SYCL has its lineage from OpenCL, it inherits the SIMT based programming model for programming the device kernel. While the SIMT model has gained its vast popularity from GPU programming, generating performant code targeting many-core processors or accelerators with traditional SIMD vector units, or SPMD-on-SIMD, remains a widely researched topic for compilers. Whole-Function Vectorization (WFV) $[1,9,14]$ and Intel's ISPC compiler [18] are both well-known efforts of SPMD-on-SIMD.

While the WFV technique proves to be effective for CPU, it does not address the unique challenge of adapting SIMT for accelerators with traditional SIMD units and requiring explicit memory hierarchy management $[4,15]$. For $\mathrm{CPU}$, as the memory subsystem brings data through the different levels of caches automatically, a single SIMD lane or SIMT thread style of optimization such as WFV suffices. On the other hand, the DaVinci architecture as shown in Figure 1, has the L1 Buffer and Unified Buffer serving as on-chip lower-level caches [19]. The exchange of data between the L1 Buffer and the Unified Buffer with external memory needs to be efficiently orchestrated. A traditional loop style vectorization working concertedly with a memory tiling [19] optimization to ensure data locality is a superior approach.

To be able to effectively apply the traditional loop style optimizations such as tiling, vectorization or parallelization, the converter thus shoulders the key task of making explicit, in other words, sinking the surrounding SYCL parallel_for loop construct into the kernel code. In summary, we make the following new contributions:

- We introduce an IR-to-IR converter to raise the abstraction of the LLVM IR to the level of the Halide IR and MLIR's Affine Dialect to effectively leverage existing loop level optimizations offered by AKG and Affine Dialect.

- We extend upon SYCL's abstraction with constructs that preserve host side and runtime side information in the LLVM IR to facilitate in the process of program reconstruction.

- We demonstrate our approach is viable with performance results.

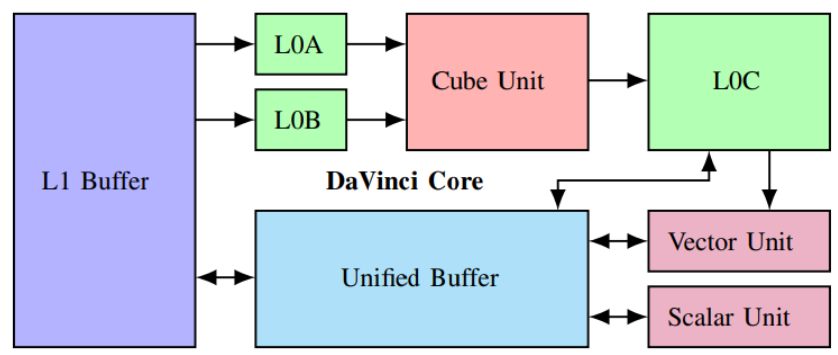

Figure 1: Overview of the DaVinci Architecture 


\section{SYCL ABSTRACTION EXTENSIONS}

Since SYCL buffers are created on the host code and kernel code, outlining happens early in the compilation process. Buffer and accessor ranges are passed as sycl: : range function arguments into the kernel code as shown in Figure 2. While sycl: : buffer creation on the host code leverages a $\mathrm{C}++$ template for the dimensionality of range $<2>(32,32)$ in line 6 of Figure 3 , the static shape information unfortunately becomes runtime constants when passed in via function arguments.

Having static shape information is important for the compiler to be able to avoid or generate efficient prolog/epilog code to handle misalignment and left-over elements. While code generation is possible with predication using runtime masking, its overhead can outweigh the benefit of using the SIMD unit, for example if the loop tripcount is small. As the LLVM IR linearizes memory accesses, recovering the shape information from the device code IR can be non-trivial [13].

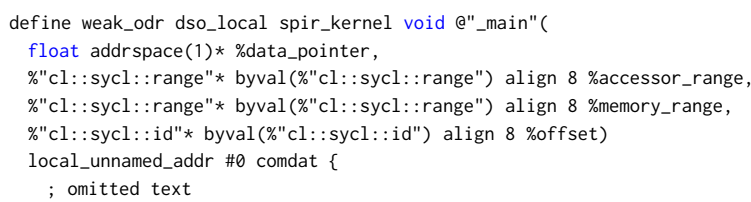

Figure 2: LLVM IR of the Kernel Code

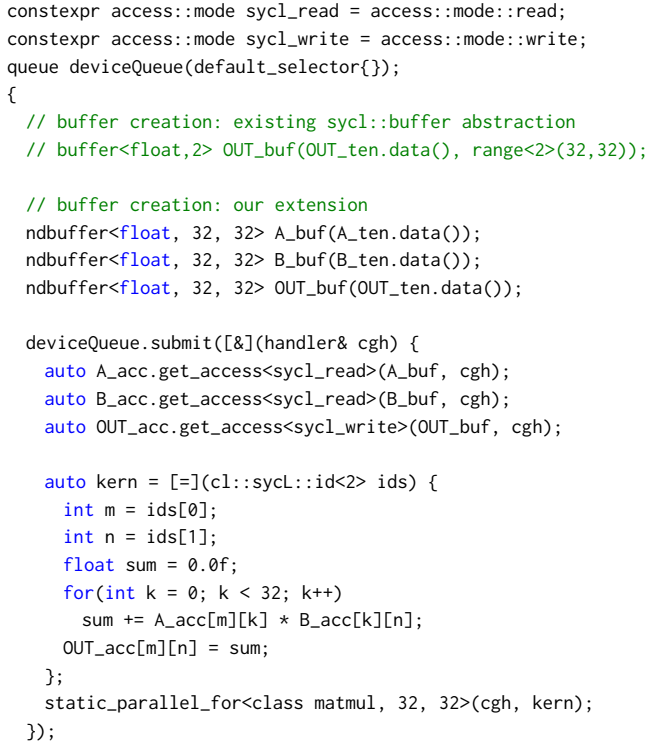

Figure 3: ndbuffer and static_parallel_for Example

To retain this information from the host code where a sycl: : buffer is first created, we create a sycl: : ndbuffer abstraction. The ndbuffer abstraction also addresses the 3D limitation inherent in sycl: : buffer.
The ndbuffer and its corresponding accessor abstraction use a $\mathrm{C}++$ variadic template and a $1 \mathrm{D}$ sycl : : buffer. Essentially, ndbuffer is a $1 \mathrm{D}$ sycl: : buffer that contains a sycl: : Array that extends the std: : array. As std: : array reveals itself as a C-struct containing a $C$ static array in the LLVM-IR, the shape information of each dimension is naturally captured by the LLVM type information in the IR of the buffer variables. We use the same technique to create the ND usmbuffer abstraction, utilizing our sycl: : Array abstraction.

To obtain the iteration space of the parallel_for construct, we need to obtain the number of work items and work groups from the SYCL runtime. When the iteration space information is statically present, we use our templated static_parallel_for or nd_parallel_for abstractions. As C++ templates embeds the template parameters into the mangled names of the spir kernel function in the LLVM IR, we can obtain the iteration space information from the kernel code's LLVM IR via demangling. The nd_parallel_for abstraction allows the user to specify parallelism in $\mathrm{n}$ dimensions rather than the limited maximum of 3D. We acknowledge that this technique currently only works if the iteration space is available during compile time.

In essence, the extended SYCL language abstractions are introduced to enrich the LLVM IR of the kernel code with extra meta-data information. Once we are successful in retaining this information in the kernel code's LLVM IR, the IR-to-IR converter processes this information into a form that later passes can consume. We refers to this process, together with raising the IR abstraction as Program Reconstruction.

\section{IR-TO-IR CONVERTER OVERVIEW}

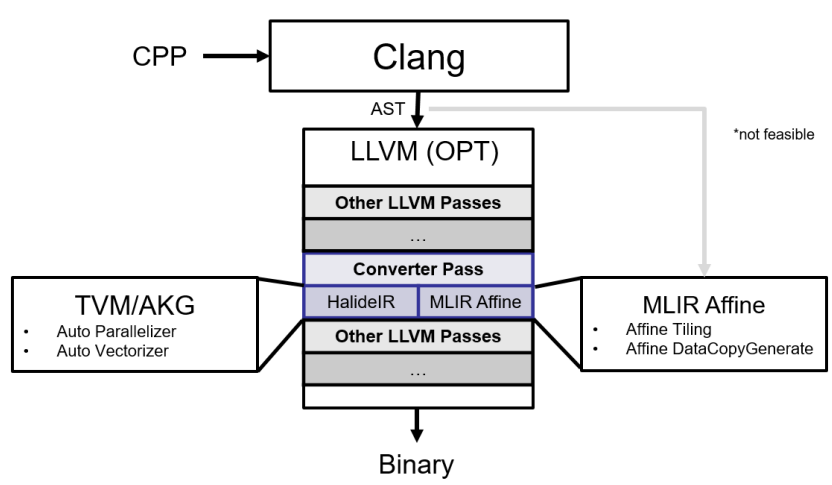

Figure 4: Compiler Flow

The IR-to-IR converter is an LLVM OPT pass as shown in Figure 4. The SYCL language abstractions are built using heavy $\mathrm{C}++$ features, particularly operator/function overloading. As such, our converter requires LLVM OPT's inlining pass to run first, in order to remove the many function calls and present clean loop and array access patterns to our converter. A direct conversion approach from the Clang AST becomes infeasible. The IR-to-IR converter has two backends: Halide IR and MLIR Affine. The DaVinci code generation currently may take either route by going through the TVM/AKG route, or MLIR's progressive lowering route. Both routes 
can be viewed as another LLVM OPT pass as the LLVM IR continues through LLC to produce the final binary.

\section{PROGRAM RECONSTRUCTION}

\subsection{Shape Reconstruction}

As described in section 2, the ndbuffer and usmbuffer abstraction embed the shape information into a chain of types in the kernel code's LLVM IR. The converter then extracts the shape information from the IR and presents it in a form that AKG can consume.

\subsection{Induction and Reduction PhiNode Identification}

LLVM IR is written in SSA form, with instructions arranged into Basic Blocks. In order to perform raising of the IR to Halide or MLIR's Affine Dialect, we must first perform various program analyses to understand the control flow and data flow of the code. Then, by traversing through the LLVM IR, we can reconstruct the high level IR constructs such as affine. for correctly.

A few analyses that are done are loop structure identification, phi node conversion, and delinearization of global indices. Rather than having Basic Blocks to deal with loops, Halide and MLIR's affine dialect both have an intrinsic for loop constructs. Halide IR is in a high-level AST form, but LLVM IR introduce the phi instructions to keep the static single assignment form (SSA). Thus we must convert phi instructions of the LLVM IR into a proper recognizable form.

Firstly, we need to identify phi instructions as the induction variables. This information is obtained by Scalar Evolution given the loop. For each induction phi variable, we introduce a scalar variable as a loop variable representing the loop index value.

Additionally, we verify that all loops are normalized. Normalized loops are loops in which the induction variable starts at 0 (or any constant) and gets incremented by one in each iteration until the exit condition is met. This means that there are no break/continues/exceptions in the loop. As such, we are able to easily extract the iteration count, and ensure that there is a single induction variable for this loop.

We also need to identify phi reduction variables. Usually, to represent a reduction, LLVM IR uses a series of phi instructions. We perform pattern matching to identify the following reduction pattern:

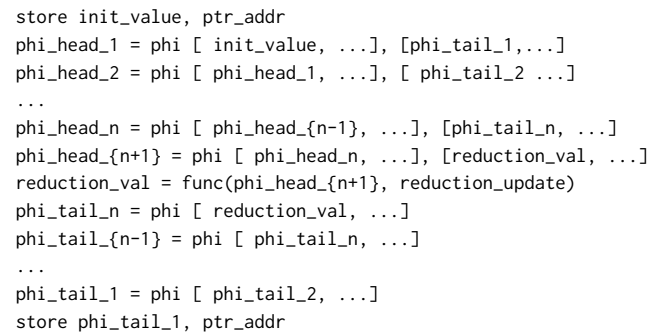

which gets recognized as the following:

13 ptr_addr $=$ init_value

14 for $(\ldots)$

15 ptr_addr $=$ func $\left(p t r \_a d d r\right.$, reduction_update $)$

Later in code generation, we skip all of the phi_head_i and phi_tail_i instructions, as well as the final store instruction

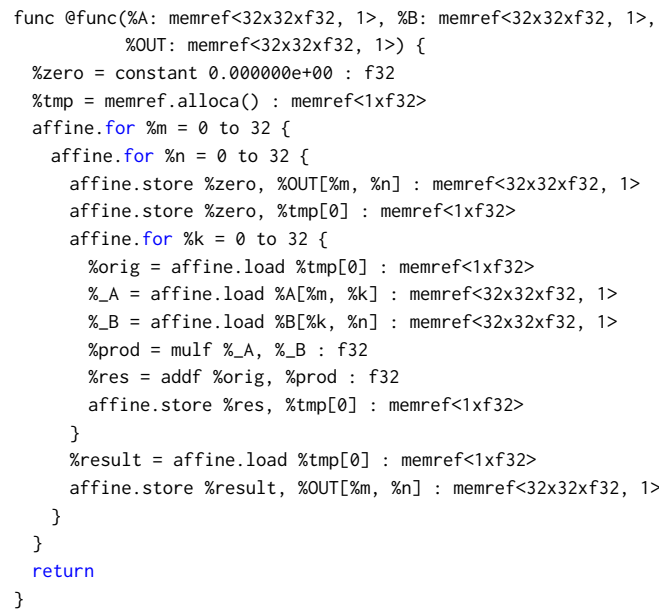

Figure 5: Output of MLIR Affine backend for matmul

on line 12 . We then generate ptr_addr on line 15 in place of reduction_value on line 7 .

Lastly, we assign a 1D tensor variable of size one to each of the non-reduction, non-induction phi variable. This results in the statement, vi[0] = incoming_value, at the end of the corresponding block. Here, vi is the tensor variable assigned to represent the phi variable.

We also perform pattern matching on delinearized global indices to accommodate our loop reconstruction pass, mapping global indices to proper variables. Once we have completed our analyses, we can generate the main body of our kernel to be embedded into our reconstructed loops.

\subsection{Iteration Space Reconstruction}

With the static_parallel_for and nd_parallel_for abstractions, the converter uses a custom demangler to extract the iteration space information. In the case of the MLIR affine backend, the converter outputs affine. for loops as shown in Figure 5 for the kernel shown in Figure 3.

During the process of sinking the parallel_for construct into the kernel code, the semantic of the code is changed from parallel execution to sequential execution. This means that if there are synchronization primitives inside the kernel body, deadlocks may occur. As such, we must activate a SemaChecker to ensure the reconstructed loops do not change the semantics of the kernel code. Data dependencies do not need to be checked but the converter issues a warning for $A[i]=A[i+1]$. Such a statement inside a parallel_for construct is an implementation error from the programmer so the converter issues a warning for the possible data race condition.

Once the converter has identified the iteration space and reconstructed the loops along with the buffer shapes, a high level form of loopy IR with attributes is given to AKG for optimization. The loopy IR enters AKG through our custom $\mathrm{C}++$ Halide IR Builder API. 


\section{MLIR PROGRESSIVE LOWERING FOR ACCELERATORS WITH AN EXPLICIT MEMORY HIERARCHY ARCHITECTURE}

\subsection{Overview: MLIR Framework}

Multi-level IR (MLIR) [7] supports different levels of abstractions of representations namely Dialects, and various transformations. It allows users to customize ops, rewrite patterns, and create Dialects targeting specific backends with extreme flexibility. Progressively lowering is needed between the different Dialects.

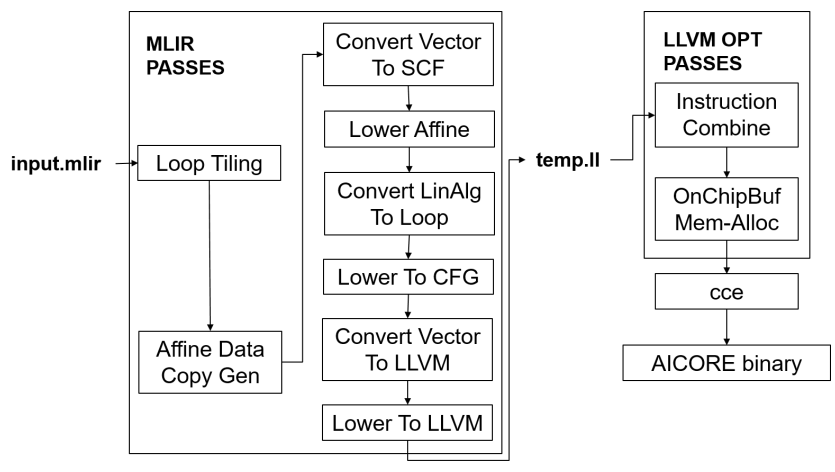

Figure 6: Passes in the mlir-llvm-compile tool

For accelerators with an explicit memory hierarchy $[4,15,17]$ architecture, software is responsible for managing data transfers between the external memory and the small on-chip buffers. Since no memory subsystem is provided by the hardware to guarantee coherency, large loops have to be broken down into smaller tiles so that data can be copied from the external memory to on-chip buffers (OnChipBuf) and vice versa, through DMA calls. In other words, tiling is no longer an optional performance optimization but an essential pass to generate functional code. As shown in Figure 6, we take advantage of the existing LoopTiling and DataCopyGeneration passes in the MLIR Affine Dialect which allow us to tile perfectly nested loops and insert DMA calls at a suitable depth level. We then progressively lower Affine Dialect to LLVM Dialect and translate LLVM Dialect into LLVM IR. An LLVM OPT pass assigns addresses to the allocated memory on the OnChipBuf. Before the OnChipBuf Mem-Alloc pass, an InstructionCombine pass is called to simplify the IR. Finally, the LLVM IR file is given to LLC of the CCE compiler to generate the final AICORE binary [12]. All the intermediate passes, from the Affine Loop Tiling and DMA generation pass in MLIR to the OnChipBuf Mem-Alloc pass in LLVM, are integrated in a single tool called mlir-llvm-compile. This tool takes in an Affine Dialect IR text as input and outputs LLVM IR which is consumed by LLC of the backend compiler.

\subsection{Affine Passes}

LoopTiling. The IR-to-IR converter described in section 3 produces a text file with the kernel code in Affine Dialect. Since the size of the OnChipBuf is a hard constraint as the data we bring into the OnChipBuf cannot exceed its capacity, the LoopTiling pass is initialized with cacheSizeBytes set to the OnChipBuf size. Our work incidentally uncovers a limitation with the existing tile size formula which takes the $\mathrm{n}^{\text {th }}$ root of the memory footprint divided by the cacheSizeBytes. When cacheSizeBytes increases, the formula erroneous results in smaller tile sizes. After LoopTiling, the inner loop may be naturally SuperVectorized. However, we defer the exploitation of vectorization as future work.

DataCopyGeneration. The current DataCopyGeneration pass in Affine Dialect performs explicit data copying. We utilize this pass to generate the necessary DMA calls. The external memory setting is the slowMemorySpace and OnChipBuf is the fastMemorySpace. By using the OnChipBuf capacity as the fastMemCapacityBytes and the cacheSizeBytes in the LoopTiling pass, we guarantee that a safe tiling strategy can be found and OnChipBuf's capacity would not be exceeded. We also customize Affine. DMA_start_cce and Affine.DMA_wait_cce to store the necessary pipe and event information needed for lowering into synchronization intrinsics later on. Corresponding ops are introduced into the Standard Dialect to support lowering.

\subsection{Lowering from Standard Dialect to LLVM Dialect}

Lowering of DMA instrinsics. There are no DMA instrinsics defined in the LLVM Dialect so direct lowering from Standard to LLVM Dialect fails. We introduce two DMA intrinsics into the LLVM Dialect which has the exact API as the CCE DMA instrinsics. To support MLIR-to-LLVM IR translation, we define the corresponding intrinsics in LLVM as well. The LLVM intrinsic signature matches that of the CCE instrinsics and new types such as void pointers to external memory space are introduced. Synchronization intrinsics are also introduced into the LLVM Dialect as the semantics of DMA calls are asynchronous. They map to the synchronization intrinsics in the LLVM IR of the CCE compiler. The synchronization instructions are placed next to the the DMA instructions.

Lowering of Alloc on OnChipBuf. The DataCopyGeneration pass in the Affine Dialect generates memory allocation and free calls in the OnChipBuf address space. Since the system or software stack of the accelerator does not provide memory management tool such as libmalloc, our tool needs to insert IR into the kernel code to manually manage the memory allocation by manipulating the address pointer for the OnChipBuf. In the Standard To LLVM conversion, we temporarily lower alloc in OnChipBuf address space to a C-like function call which is later processed by the LLVM OnChipBuf Mem-Alloc OPT pass.

\subsection{LLVM Passes}

OnChipBufMemoryAllocation. After obtaining the LLVM IR from translateModuleToLLVMIR, we run the InstructionCombine pass and then the OnChipBufMem-Alloc pass to clean up the IR. A Clike function which is used to represent memory allocation in the OnChipBuf address space, created in the Standard to LLVM conversion pass, are searched and turned into an IntToPtr instruction. A simple memory allocation mechanism is implemented where memory allocation corresponds to a pointer increment and memory free corresponds to a pointer decrement. 


\subsection{Integrity of the Original MLIR Lowering Pipeline}

Our custom lowering pipeline with modifications to LoopTiling, DataCopyGeneration passes and Standard To LLVM conversion are currently guarded using the module target triple attribute as the flag. This helps to preserve the original MLIR lowering pipeline for other platforms.

\section{EXPERIMENTS}

To verify that our IR-to-IR Converter with the MLIR backend, shown in Figure 4, can work for a CPU backend, we use MLIR's original lowering pipeline to generate the LLVM IR. The LLVM IR is then compiled with $-03-\mathrm{ffast}-$ math $-\mathrm{std}=\mathrm{c}++17$ to produce an ARM64 executable and ran on Huawei's Kunpeng 920 server. Our SYCL implementation is based on sycl-nightly/20210514 [6], with LLVM 13.

The clock speed of the server is $2.6 \mathrm{GHz}$ with $64 \mathrm{~KB} \mathrm{L1}$ data cache, 512 KB L2 cache and 24M L3 cache. We measure the speed of double precision Matrix Multiplication kernels where $\mathrm{M}=\mathrm{N}=\mathrm{K}$ in GFlops. We take an average of 10 runs with max and min values removed. We compare our MLIR path with that of Eigen3.3 [5] with and without OpenBLAS [8], with OPENBLAS_NUM_THREADS=1. We run the experiments with different matrix sizes ranging from 40 to 3100 . The compiler options for Eigen is $-03-f f a s t-m a t h-s t d=c++17$ $-m a r c h=a r m v 8-a-m f p u=v f p v 3$. As shown in Figure 7, Eigen with OpenBLAS has the best performance reaching 10GFlops which is almost the single core theoretical peak. Our MLIR path only achieves 1 to 2 GFlops with cacheSizeBytes set to $1 \mathrm{~K}$ or $64 \mathrm{~K}$. The reason is that only Affine LoopTiling pass with the default tiling strategy is utilized to tile the M,N,K loops equally. Performance critical optimizations such as packing, register tiling and data prefetching for the micro-kernel are not performed.

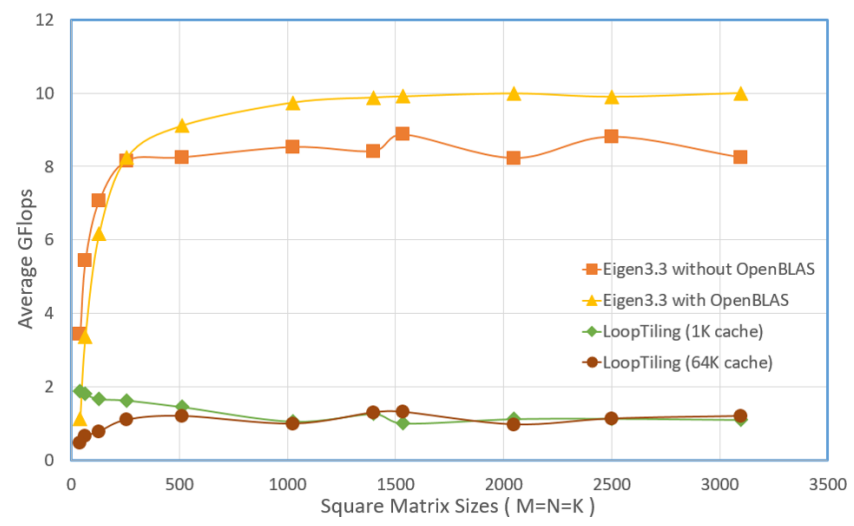

Figure 7: MLIR Code Generation: Performance Comparison with Eigen 3.3 and OpenBLAS

We also compare performance on DaVinci using our customized AICORE MLIR lowering pipeline with that of using AKG via the Halide IR backend. We measure using Huawei's Ascend 910 Server with 32 AICORES but we focus on measuring a single core performance using hardware performance counters. Both paths do not make use of the cube unit. We set the cacheSizeBytes and fastMemorySpace to be $256 \mathrm{~K}$, matching the size of the Unified Buffer.

We show speedup comparison using five kernels: ReLU 16x32, ReLU 32x32, MatMul 16x16x16, MatMul 32x32x32, and MatMul 64x64x64. As shown in Figure 8, 3.02 to 20.58 times speedups are achieved by AKG's code generation.

Distribution of cycles spent in executing scalar, vector and memory instructions are gathered through hardware performance counters and presented in Figure 9. Since AICORE's scalar unit is significantly slower than its vector unit, the ability to use vector instructions in AKG contributes to its performance advantage. The speedup can also be attributed to AKG's usage of a polyhedral analysis for tiling in combination with an auto-search strategy. AKG also performs double buffering to overlap DMA transfers with computation as well as inserting the required DMA and synchronization instructions based on dependency analysis. Thus, AKG is a much more mature optimizer than our current MLIR lowering pipeline. The ReLU kernels are less computationally intensive as compared to matrix multiplication kernels and therefore, higher percentage of memory instructions are observed.

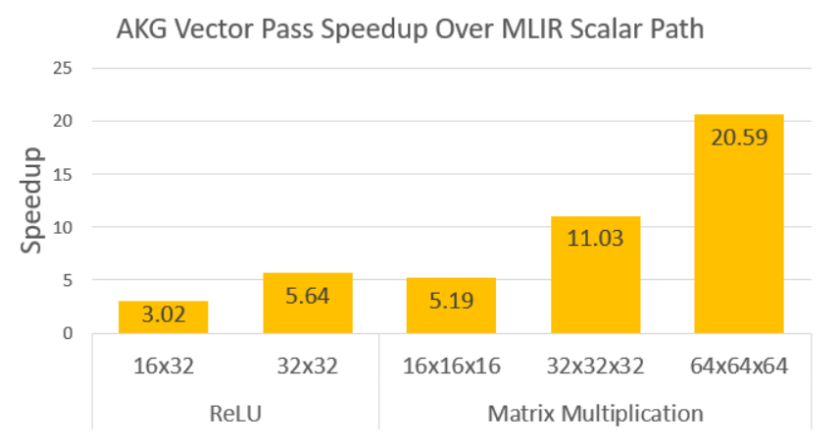

Figure 8: Performance Speedup using AKG

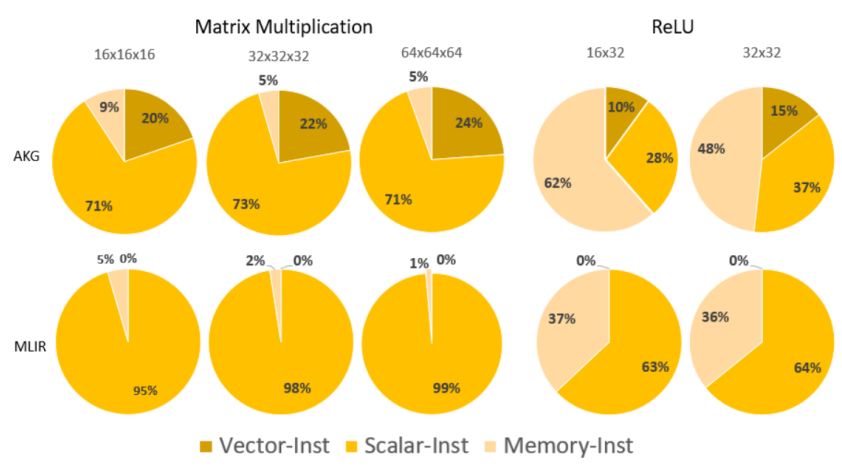

Figure 9: Distribution of Execution Cycles 


\section{RELATED WORK}

Moses and Chelini's work $[11,16]$ raises the IR abstraction using the MLIR Extraction (MET) tool for C code and enters the Affine Dialect directly from Clang's AST. There is also the CIL project which compiles $\mathrm{C}++/ \mathrm{C}$ into the $\mathrm{CIL}$ [3] Dialect directly from Clang.

\section{CONCLUSION}

We have implemented an IR-to-IR converter which raises the LLVM IR to a higher abstraction level for the purpose of generating efficient SYCL kernel code written with the SIMT programming style. We would like to explore more MLIR passes to close the performance gap between MLIR and AKG in the future.

\section{ACKNOWLEDGMENTS}

We would like to acknowledge the contributions from our colleagues: Yaoqing Gao, Wilson Feng, Rasool Maghareh, and Giancarlo Colmenares. We also wish to thank the anonymous reviewers for their help in improving the quality of the paper.

\section{REFERENCES}

[1] 2015. LLVM Developers' Meeting: Pierre-Andre Saulais "Creating an SPMD Vectorizer". https://www.youtube.com/watch?v=ePu6c4FLc9I.

[2] 2021. Automatic Kernel Generator. https://gitee.com/mindspore/akg/.

[3] 2021. C Language Family Front-end. https://github.com/compiler-treetechnologies/cil/tree/cil/clang/.

[4] 2021. Cambricon MLU Hardware Architecture. https://www.cambricon.com/ docs/bangc/developer_guide_html/3MLUhardwarearchitecture/index.html.

[5] 2021. Eigen 3.3.9. https://eigen.tuxfamily.org/.
[6] 2021. Intel's LLVM Project. https://github.com/intel/llvm/.

[7] 2021. Multi-Level IR Compiler Framework. https://mlir.llvm.org/.

[8] 2021. An Optimized BLAS Library. https://www.openblas.net/.

[9] 2021. The Region Vectorizer (RV). https://github.com/cdl-saarland/rv.

[10] 2021. SYCL Khronos Group. https://khronos.org/sycl/.

[11] Lorenzo Chelini, Andi Drebes, Oleksandr Zinenko, Albert Cohen, Nicolas Vasilache, Tobias Grosser, and Henk Corporaal. 2021. Progressive Raising in Multilevel IR. In 2021 IEEE/ACM International Symposium on Code Generation and Optimization (CGO). 15-26. https://doi.org/10.1109/CGO51591.2021.9370332

[12] Wilson Feng, Rasool Maghareh, and Kai-Ting Amy Wang. 2021. Extending DPC++ with Support for Huawei Ascend AI Chipset. In International Workshop on OpenCL (Munich, Germany) (IWOCL'21). Association for Computing Machinery, New York, NY, USA, Article 13, 4 pages. https://doi.org/10.1145/3456669.3456684

[13] Tobias Grosser, Sebastian Pop, J Ramanujam, and P Sadayappan. 2015. On Recovering Multi-Dimensional Arrays in Polly. http://impact.gforge.inria.fr/ impact2015/index.html Fifth International Workshop on Polyhedral Compilation Techniques, Impact 2015 ; Conference date: 19-01-2015 Through 19-01-2015.

[14] Ralf Karrenberg and Sebastian Hack. 2011. Whole-function vectorization. In International Symposium on Code Generation and Optimization (CGO 2011). 141150. https://doi.org/10.1109/CGO.2011.5764682

[15] Heng Liao, Jiajin Tu, Jing Xia, and Xiping Zhou. 2019. DaVinci: A Scalable Architecture for Neural Network Computing. In 2019 IEEE Hot Chips 31 Symposium (HCS). 1-44. https://doi.org/10.1109/HOTCHIPS.2019.8875654

[16] William S. Moses, Lorenzo Chelini, Ruizhe Zhao, and Oleksandr Zinenko. 2021. Polygeist: Affine $C$ in MLIR. https://acohen.gitlabpages.inria.fr/impact/ impact2021/papers/IMPACT_2021_paper_1.pdf Not a formal proceedings.

[17] Maurice Peemen, Bart Mesman, and Henk Corporaal. 2015. Inter-Tile Reuse Optimization Applied to Bandwidth Constrained Embedded Accelerators. In Proceedings of the 2015 Design, Automation \& Test in Europe Conference \& Exhibition (Grenoble, France) (DATE '15). EDA Consortium, San Jose, CA, USA, 169-174.

[18] Matt Pharr and William Mark. 2012. ispc: A SPMD compiler for high-performance CPU programming. 2012 Innovative Parallel Computing, InPar 2012, 1-13. https: //doi.org/10.1109/InPar.2012.6339601

[19] Jie Zhao and Peng Di. 2020. Optimizing the Memory Hierarchy by Compositing Automatic Transformations on Computations and Data. In 2020 53rd Annual IEEE/ACM International Symposium on Microarchitecture (MICRO). 427-441. https: //doi.org/10.1109/MICRO50266.2020.00044 